\title{
Review Article on Designing of Pseudo Quadratue Mirror Filter Bank
}

\author{
Shaheen ${ }^{1}$, Ankita Gupta ${ }^{2}$ \\ ${ }^{I}$ (Electronics and Communication department, Student of Rajasthan College of Engineering for Women, India) \\ ${ }^{2}$ (Electronic and Communication department, Assistant professor, Poornima Group of Institution, India)
}

\begin{abstract}
This paper presents the review of the Pseudo-QMF bank which is one of the most important subclass of the Multi-rate filter banks. It deals with the advantages of Pseudo-QMF bank over the other filter banks such as fewer complexes in designing and easy to implement. Various design techniques of pseudo-QMF have been discussed here, so that this paper would help other researchers to work in this very important field of digital signal processing.
\end{abstract}

Keywords: Cosine modulated filter bank, Kaiser window approach (KWA), Pseudo-QMF, Non-uniform subband filter bank, Spectral factorization approach.

\section{Introduction}

It is found in many signals that their energy is dominantly concentrated in a particular region of frequency. To save the bandwidth in such signals, signal can be compressed and decimated. But simple way of compression introduces aliasing in the signal. So Quadrarue Mirror Filter (QMF) comes as a solution to this problem as it saves the bandwidth and increases efficiency without compromising the quality of the signal. QMF banks are used in image processing, antenna systems, subband coding of speech signal, transmultiplexer in telecommunication, in digital audio industry and in biomedical engineering.

Pseudo-QMF is a subclass of the QMF bank which further belongs to the large family of multi-rate filter bank. Pseudo-QMF basically belongs to the family of cosine modulated filter banks. A type of filter bank in which the distortion is approximately a delay and the aliasing is canceled approximately is known as pseudoQMF bank. Pseudo-QMF bank gradually becomes popular in the field of signal processing because of its many attractive features such as easy to design; more realizable, more efficient with low cost, number of parameters to optimize is less due to prototype filter design.

A signal may be separated into two or more sub-band with the help of QMF so that each band signal can be processed independently to achieve sufficient compression of signal without compromising the quality of the signal. Then these sub-band signals are recombined to get the original signal back [1].

Orthogonal filter banks and biorthogonal banks are the major classification of the quadrature mirror filter banks [2]. All of these filters can be designed using either the near perfect reconstruction (NPR) or perfect reconstruction (PR) [3]. Orthogonal filters are always created using nonlinear-phase filters while either linear phase or nonlinear-phase can be used for designing of QMF banks and biorthogonal filter banks [4].

One of the most frequently used filter bank among all the M-band filter banks is cosine-modulated filter bank (CMFB) because the designing is easier and more realizable than that of other filter banks $[3,6]$. In CMFB all the filters are cosine modulated version of a low-pass prototype filter. So the design of whole filter bank reduces to the design of a single prototype filter. Implementation of CMFB consists of one prototype and a discrete cosine transform (DCT). Near perfect reconstruction (NPR) finite impulse response (FIR) CMFB avoids computation of large matrix sets. There are two types of CMFB one is with perfect reconstruction [7] and the other is pseudo-QMF [8]. Unlike PR filter banks, in pseudo-QMF aliasing is canceled approximately and the distortion is approximately a delay $[3,6]$ and the approximation improves with the filter order.

Fig.1. shows the typical two channels QMF bank and Fig.2. shows the frequency response of this filter. This filter splits the signal into two sub-bands using high-pass and low-pass filters $\mathrm{H}_{1}(\mathrm{Z})$ and $\mathrm{H}_{0}(\mathrm{Z})$ respectively. These signals are decimated, coded and transmitted on the transmitter side. These signals are decoded, interpolated and then finally passed though the filters to recombine the signals. The reconstructed signal is not exact replica of the transmitted signal, it suffers from three types of distortions: amplitude distortion (AMD), phase distortion (PHD) and aliasing distortion (ALD). 


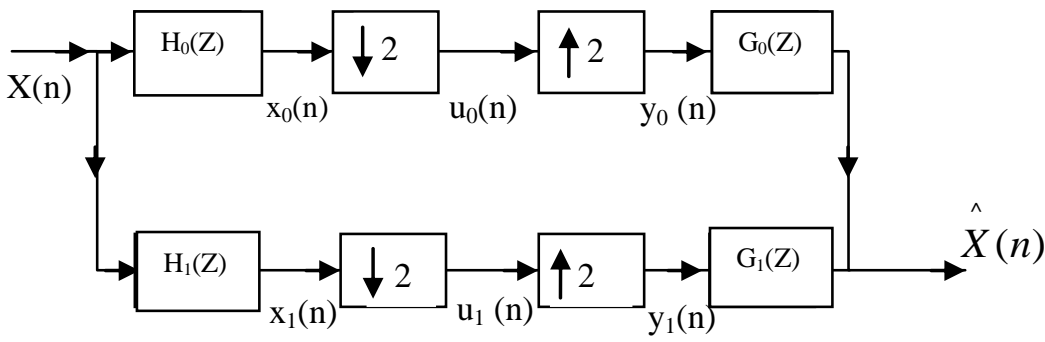

Figure.1. Two channel QMF

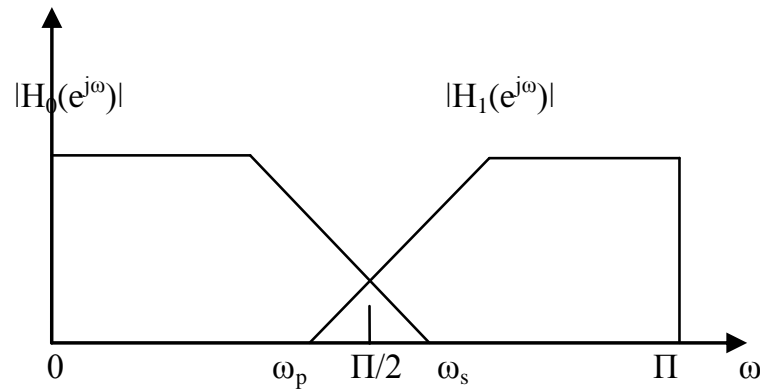

Most of the researchers stresses on the elimination or minimization of these distortions to obtain the perfect reconstruction (PR) or near perfect reconstruction (NPR) system [4].

Pseudo-QMF bank is used in speech enhancement, image processing, sub-band coding, trans-multiplexer and in biomedical engineering.

This paper is organized as follows. This section gives the brief introduction of pseudo-QMF, analysis is given in the section 2 while section 3 deals with the design techniques. Finally section 5 gives the conclusion.

\section{Analysis Of The Pseudo-Qmf Bank}

In pseudo-QMF bank all the analysis and synthesis filters are the cosine modulated version of the low pass prototype filter. Pseudo-QMF bank was first proposed by Nussbaumer [6] and Rothweiler [9], Chu developed the various approaches to design these filter banks [10]

$U_{0}\left(e^{j \omega}\right)=\left[X_{0}\left(e^{j \omega / 2}\right)+X_{0}\left(-e^{j \omega / 2}\right)\right] / 2$

The above equation is formed using the input output relation of decimator and interpolator which gives the aliasing effect in channel and (2) gives the imaging effect.

$Y_{0}\left(e^{j \omega}\right)=U_{0}\left(e^{2 j \omega}\right)$

The relation between $\mathrm{Y}(\mathrm{Z})$ and $\mathrm{X}(\mathrm{Z})$ of two channel QMF bank can be written as

$Y(Z)=1 / 2\left[H_{0}(Z) G_{0}(Z)+H_{1}(Z) G_{1}(Z)\right] X(Z)+1 / 2\left[H_{0}(-Z) G_{0}(Z)+H_{1}(-Z) G_{1}(Z)\right] X(-Z)$

(3)

Second term of (3) represents aliasing. It can be removed by wisely choosing the synthesis filter according to the analysis filter as conditions given in (4) below:

$G_{0}(Z)=H_{1}(-Z), G_{1}(Z)=H_{0}(-Z)$ and $H_{1}(Z)=H_{0}(-Z)$

On further simplification we get

$G_{0}(Z)=H_{0}(Z)$ and $G_{1}(Z)=-H_{1}(Z)=H_{0}(-Z)$

All the prototype filter can be designed using the low pass analysis filter $\mathrm{H}_{0}(\mathrm{Z})$ only.

Cosine modulation filter bank is a kind of filter bank, it is very popular because of ease of designing and its simplicity. All filters can be designed in this filter bank by cosine modulation of low-pass prototype filter. Modulated filter banks can be designed either using either PR technique or NPR technique [5]. Pseudo QMF designs are more attractive because of ease in designing and it gives more selective prototype filter in comparison to the PR. The overall transfer function should be equal to simple approximately a delay to give the result pseudo-QMF design. 


\section{Design Techniques Of Pseudo-Qmf}

CMF banks have been derived from DFT filter banks as follows:

a) $2 \mathrm{M}$ complex modulated filters are used in both analysis and synthesis filter banks.

b) The bandwidth of the prototype filter is $\pi / 2 M$.

c) Channel filters are derived by complex modulation of prototype filter at frequencies $(2 \mathrm{i}+1) / 2 \mathrm{M}$.

d) Real impulse of the channel filters are obtained by adding the impulse responses of the complex conjugates of the channel filter which occurs in pairs.

J. Kliewer shown in [11] that pseudo-QMF is just a special case of the perfect reconstruction case. So the pseudo-QMF prototype can be designed using the perfect reconstruction method. In fact complete framework of perfect reconstruction can also be used for the pseudo-QMF case.

There have been many designing techniques for CMFB $[6,10,12]$ but they were not computationally efficient. Then Creusere and Mitra [13] have introduces a new techniques and there conditions are given as

$$
\begin{aligned}
& \left|H_{0}\left(e^{j \omega}\right)\right|^{2}+\left|H_{0}\left(e^{j(\omega-\pi / M)}\right)\right|^{2}=1 \text { for } 0<\omega<\pi / M \\
& \left|H_{0}\left(e^{j \omega}\right)\right|=0 \text { for } \omega>\pi / M
\end{aligned}
$$

Where, $M$ is the number of bands and , $\omega$ is the frequency.

$\mathrm{g}_{\mathrm{k}}(\mathrm{n})$ and $\mathrm{h}_{\mathrm{k}}(\mathrm{n})$ are the impulse responses of the analysis and synthesis filters and they are the cosine modulated versions of the prototype filter $\mathrm{h}(\mathrm{n})[14]$ and is given by

$\mathrm{h}_{\mathrm{k}}(\mathrm{n})=2 \mathrm{~h}(\mathrm{n}) \cos \left((2 \mathrm{k}+1) \cdot \frac{\Pi}{2 \mathrm{M}} \cdot\left(\mathrm{n}-\frac{\mathrm{N}-1}{2}\right)+(-1)^{\mathrm{k}} \cdot \pi / 4\right)$

$\mathrm{g}_{\mathrm{k}}(\mathrm{n})=2 \mathrm{~g}(\mathrm{n}) \cos \left((2 \mathrm{k}+1) \cdot \frac{\Pi}{2 \mathrm{M}} \cdot\left(\mathrm{n}-\frac{\mathrm{N}-1}{2}\right)-(-1)^{\mathrm{k}} \cdot \pi / 4\right)$

Where, $\mathrm{M}$ is the number of bands.

The overall transfer function is given by [15]

$$
\begin{aligned}
T_{0}(Z) & =1 / M \sum_{k=0}^{M-1} H_{k}(Z) G_{k}(Z) \\
= & z^{-(N-1)} / M \sum_{k=0}^{M-1} H_{k}(Z) \tilde{H}_{k}(Z)
\end{aligned}
$$

Since $T_{0}(Z)$ has a linear phase independent of $H_{k}(Z)$;so the reconstructed signal has no phase distortion. It has been shown in [16] if (6) is evaluated at $\omega=\Pi / 2 \mathrm{M}$ then we achieve a new equation which gives a new PR condition shown below. Amplitude response (AR) remains 0.707 in this case.

$$
\begin{aligned}
& \left|H_{0}\left(e^{j \omega / 2 M}\right)\right|^{2}+\left|H_{0}\left(e^{j(\pi / 2 M-\pi / M)}\right)\right|^{2}=1 \\
& \left|H_{0}\left(e^{(j \pi / 2 M)}\right)\right|=0.707
\end{aligned}
$$

This new PR condition is satisfied by optimizing pass-band frequency. This approach gives low ALD with less number of iterations.

J.Kliewer and A. Mertines shown in [17] that oversampled cosine modulated filter bank can be used in making modification in certain frequency bands of signal.

Y.P. Lin and P.P. Vaidyanathan [18] has introduced a method to design maximally decimated FIR cosine modulated filter bank with analysis and synthesis filters both in linear phase. This technique can be used either in perfect reconstruction or in pseudo-QMF case.

In this technique two filter bank has the bandwidth double of that of the other filters. The filter banks are divided into two subsystems, the first one has half plus one channels and second one has half minus one channels. Aliasing components are cancelled because of spectral overlapping in first and second subsystem.

Result is alias free with flat magnitude and phase responses. This technique provides better coding gain and good attenuation characteristics at the reasonable cost.

Koilpillai and Vaidyanathan introduced spectral factorization approach in [19] in which prototype filter is obtained by spectral factorization of a $2 \mathrm{M}^{\text {th }}$ band filter which makes the designing of prototype filter easier by eliminating the need of any optimization. This approach introduces the cancelation of all the significant aliasing term. So this approach present the alias free reconstruction and a flat response of the overall transfer function of the analysis/synthesis filter.

J.Princen [20] presents an approach to design a non-uniform filter bank in which uniform sections are joined by transition filters to generate non-uniform filter banks. Aliasing cancellation is accomplished by designing transition filter by modulating complex low-pass prototype filter. 
Alternate analysis and synthesis filter (AASF) [21] is an approach to the designing of prototype filter for the pseudo-QMF bank. In this approach analysis and synthesis filters are obtained by simply modulating the linear phase type I or type II FIR filter. AASF approach introduces very low amplitude distortion, doesn't have any phase distortion and aliasing error is present in the tolerable range and its designing is comparatively easy.

Combinational window function with high side-lobe-fall-off-rate (SLFOR) presented in [22] introduces filter banks with very low reconstruction error and aliasing error at low cost. These filter banks can be used in echo cancellation and cross-talk suppression.

S. Jayasimha and C.G.Hiremath [23] modified the equiripple filter to reduce the maximum overall reconstruction error by slightly compromising the equiripple property of the filter. This technique gives the result with linear-phase spectral factor.

Tree structured [24] method is an easy and simple method of obtaining non-uniform filter banks. But it has the problem of restricted sampling rate and non optimal frequency response.

Non-uniform filter bank with integer sampling is introduced by J. Li, T.Q. Nguyen and S.Tantaratana [25]. This technique is much simpler than the other filter bank design techniques. In this technique non-uniform filter banks are designed using the uniform cosine modulated filter banks. Amplitude distortion and aliasing distortion are very small and phase distortion is zero in the result obtained with this technique.

Deng, Mathews and Boroujeny introduced a non-uniform pseudo QMF bank with low delay [26]. A number of band of low delay uniform banks are combined together to form the non-uniform delay pseudo-QMF bank. It provides sharp transition band, high stop-band attenuation, low delay and low aliasing error. It also solves the problem of frequency localization.

A.Jain and A.P.Goel has introduced particle swarm optimization method [27] to design pseudo-QMF bank for image coder. Result obtained with this technique shows very low aliasing, higher coding gain, zero PHD and low ALD. It also improves the visual quality of the image.

K.Shao [28] introduced the modified Fourier transform (MDFT) filter banks which is based on pseudoQMF filter bank and gives nearly perfect reconstruction (NPR). MDFT is used in multicarrier modulation systems. On comparing with OFDM, it shows that MDFT is better than that of the OFDM.

Table 1

Errors introduced by different designing techniques of pseudo-QMF banks

\begin{tabular}{|c|c|c|c|c|}
\hline Pseudo-QMF bank & Prototype filter design method & PHD & AMD & ALD \\
\hline $\begin{array}{l}\text { SFA (Spectral } \\
\text { Factorization Approach) }\end{array}$ & $\begin{array}{l}\text { Any technique to design spectral } \\
\text { factor of linear-phase } 2 \mathrm{M}^{\text {th }} \text { band } \\
\text { filters }\end{array}$ & $\mathrm{NO}$ & $\begin{array}{l}\text { It can be } \\
\text { high at } \omega=0 \\
\text { and } \omega=\pi\end{array}$ & $\begin{array}{l}\text { Slightly higher than } \\
\text { stopband attenuation of } \\
\text { the prototype filter }\end{array}$ \\
\hline $\begin{array}{l}\text { KWA (Kaiser Window } \\
\text { approach) }\end{array}$ & $\begin{array}{l}\text { Through optimization, using a } \\
\text { Kaiser window }\end{array}$ & NO & Very low & $\begin{array}{l}\text { Slightly higher than } \\
\text { stopband attenuation of } \\
\text { the prototype filter }\end{array}$ \\
\hline $\begin{array}{l}\text { AASF } \\
\text { Analysis and } \begin{array}{r}\text { Synthesis } \\
\text { Filters) }\end{array}\end{array}$ & $\begin{array}{l}\text { Any linear-phase FIR technique } \\
\text { capable to control the position of } \omega_{c}\end{array}$ & $\mathrm{NO}$ & Very low & $\begin{array}{l}\text { Slightly higher than } \\
\text { stopband attenuation of } \\
\text { the prototype filter }\end{array}$ \\
\hline $\begin{array}{l}\text { NPR (Near } \quad \text { Perfect } \\
\text { Reconstruction) }\end{array}$ & Through optimization & NO & Almost null & $\begin{array}{l}\text { Similar to stopband } \\
\text { attenuation of the } \\
\text { prototype filter }\end{array}$ \\
\hline Low-delay Non-uniform & $\begin{array}{l}\text { Through combining bands of } \\
\text { uniform pseudo-QMF }\end{array}$ & NO & Very low & Very low \\
\hline $\begin{array}{l}\text { Linear Phase } \mathrm{CM} \\
\text { maximally decimated }\end{array}$ & Through optimization technique & NO & Very low & NO \\
\hline $\begin{array}{l}\text { Integer sampling non- } \\
\text { uniform filter banks. }\end{array}$ & Through optimization & $\mathrm{NO}$ & Very low & Very low \\
\hline $\begin{array}{l}\text { Particle swarm } \\
\text { optimization approach }\end{array}$ & Through any optimization technique & $\mathrm{NO}$ & Low & Very low \\
\hline
\end{tabular}

Table 1 show the various techniques being used for the designing of pseudo-QMF bank and compares various errors presents in the pseudo-QMF banks to give the better view of each technique.

\section{Conclusion}

We have discussed pseudo-QMF bank in this paper and got to know that how pseudo-QMF is better than other types of filter banks in many aspects. Pseudo-QMF is easy to design and is more realizable, that's why it is more popular than the other filter banks. We have discussed various design techniques and compared them which may be very useful for the researchers.

\section{References}

[1] R. E. Crochiere, "Sub-band coding," The Bell System Technical Journal, vol. 60, no. 7, pp. 1633-1653, 1981. 
[2] R. Bregovic and T. Saramaki, "A general-purpose optimization approach for designing two-channel fir filterbanks," IEEE Transactions on Signal Processing, vol. 51, no. 7, pp. 1783-1791, 2003

[3] P. P. Vaidyanathan, "Multirate Systems and Filter Banks", Prentice Hall, Englewood Cliffs, NJ, USA, 1993.

[4] R. Bregovic and T. Saramaki, "Two-channel FIR filterbanks-a tutorial review and new results," in Proceedings of the $2^{\text {nd }}$ International Workshop on Transforms Filter banks, vol. 4, pp. 507-558, Brandenburg, Germany, 1999.

[5] J org Kliewer, "On the Relationship between Pseudo-QMF Designs and Perfect-Reconstruction Solutions for Modulated Filter Banks", IEEE . 52 (9) (2004) 2661-2664

[6] J. H. Nussbaumer and M. Vetterli, "Pseudo quadrature mirror filters," in Proceeding Conference DSP Florence 81, pp. 8-12 (1981).

[7] T. A. Ramstad, "Cosine modulated analysis-synthesis filter banks with critical sampling and perfect reconstruction," in Proc. IEEE Int. Con\$ Acoust., Speech Signal Processing, Toronto, Canada, May 1991, pp. 1789-1792.

[8] R. V. Cox, 'The design of uniformly and nonuniformly spaced pseudo QMF," IEEE Trans. Acoust., Speech Signal Processing, vol. ASSP-34, pp. 1090-1096, Oct. 1986

[9] Rothweiler, J. H., Polyphase quadrature filters-A new subband coding technique. In Proc. Of International Conference on ASSP'83, Boston, Vol. 41, 1983, pp. 1280-1283.

[10] P. L. Chu, " Quadrature mirror filter design for an arbitrary number of equal bandwidth channels ," IEEE transaction on acoustic, speech, signal processing, vol. ASSP 33, no. 1, pp. 203-218, 1985.

[11] J. Kliewer, "On the relationship between pseudo-QMF design and perfect-econstruction solutions for modulated filter banks", IEEE, 7803-5700, 1999.

[12] T. Q. Nguyen, "Near perfect reconstruction pseudo QMF, "IEEE transaction on signal processing," vol. 42, no.1, pp.65-76, 1994

[13] C. D. Creusere, and S. K. Mitra, "A simple method for designing high quality prototype filters for M-band pseudo QMF banks," IEEE Transactions on Signal Processing," vol. 43, pp. 1005-1007, 1995.

[14] R.K. Soni, A. Jain and R. Saxena, “ Design of NPR-Type Cosine Modulated Filterbank Using Combinational Window Functions”, IJCNSS. 2010, 3, 934-942.

[15] T.Q. Nguyen, "Near-perfect-reconstruction pseudo-QMF banks", IEEE, 1053-587X, 1994

[16] A. Kumar, G.K. Singh and R.S. Anand, "An improved method for designing prototype filter for M-band pseudo QMF banks", ICCET, 2009.

[17] J.Kliewer and A. Mertins, "Design of paraunitary oversampled cosine modulated filter banks", IEEE Trans. Acoust., Speech Signal Processing, 2073-2076, 1997.

[18] Y-P. Lin and P. P. Vaidyanathan, "Linear phase cosine modulated maximally decimated filter banks with perfect reconstruction", IEEE Transaction on signal processing, 1053-567X, 1995.

[19] R.D.Koilpillai and P.P. Vaidyanathan, "A spectral factorization approach to pseudo-QMF design", IEEE,2000.

[20] J.Princen, "The design of nonuniform modulated filterbanks", IEEE, 1053-587X, 1995.

[21] F.Cruz-Roldan, P.Amo-Lopez, P.Martin-Martin and F.Lopez-Ferreras, "Alternating analysis and synthesis filters: A new pseudoQMF bank", Academic press, 329-345, 2001.

[22] A.Jain, R.Saxena and S.C.Saxena, "An improved and simplified design of cosine-modulated pseudo-QMF filter banks", ELSEVIER, 225-232, 2006.

[23] S. Jayasimha and C. G. Hiremath, "Pseudo-QMF banks near-equiripple performance”, IEEE, 1053-587X, 1998.

[24] P. P. Vaidyanathan, "Multirate Systems and Filter Banks", Englewood Cliffs, NJ, Prentice-Hall, 1993.

[25] J. Li, T. Q. Nguyen and S. Tantaratana, "A simple design method for near-perfect-reconstruction nonuniform filter banks", IEEE Transaction on signal processing, Vol. 45, NO. 8, Aug. 1997

[26] Y.Deng, V.J.Mathews and B.Farhang-Boroujeny, "Low-delay nonuniform pseudo-QMF banks with application to speech enhancement", IEEE, 2007.

[27] A. Jain and A. P. Goel, "Design of M-channel pseudo near perfect reconstruction QMF bank for image compression", Signal \& Image Processing : An International Journal (SIPIJ), Vol.3, No.4, August 2012.

[28] K.Shao, "Design and implementation of MDFT filter bank based multicarrier modulation systems", IEEE, 1389-8992, 2013. 